\title{
SISTEMAS OPEN BIM PARA FABRICANTES
}

\author{
Paula Assis ${ }^{(1)}$, Ricardo Figueira ${ }^{(2)}$, Pablo Gilabert ${ }^{(3)}$ \\ (1) Top Informática, Lda., Braga \\ (2) Top Informática, Lda., Braga \\ (3) CYPE Ingenieros, S.A., Alicante
}

\begin{abstract}
Resumo
A implementação da metodologia Open BIM tem, entre outros, o objetivo de facilitar os processos de comunicação entre os agentes intervenientes no projeto. A tecnologia Open BIM implementada no software CYPE bem como a plataforma BIMserver.center, destinada à partilha de informação para projetos de arquitetura, engenharia e construção, possibilitam o desenvolvimento de um fluxo de trabalho colaborativo em Open BIM que permite realizar projetos de forma aberta entre os distintos agentes, entre os quais se consideram os próprios fabricantes de materiais e equipamentos. O modelo federado do projeto encontra-se disponível, na plataforma BIMserver.center, para todos os intervenientes e representa em cada momento o estado de avanço do projeto para cada uma das fases do seu ciclo de vida. Neste trabalho foi criado o procedimento para a conceção dos Open BIM Systems, ou seja, sistemas Open BIM para fabricantes e foi implementado para várias marcas. Os Open BIM Systems são materializados numa aplicação para cada tipologia de produto do fabricante, disponível para projetistas e fabricante, com a capacidade de modelar, calcular, dimensionar, com base em critérios normativos, e de posicionar o produto no modelo federado do projeto. Os fabricantes são convidados ou propõem-se participar no projeto, apresentando a solução que considerem adequada face às especificações do projeto. Apresentam-se dois casos de estudo para dois fabricantes que evidenciam os resultados alcançados com os respetivos Open BIM Systems, concebidos para as duas marcas em questão, e que são representativos dos restantes Open BIM Systems.
\end{abstract}

\section{Introdução}

O modelo digital aspira a ser uma representação virtual realista do edifício. Um dos fatores mais importantes para que o modelo 3D seja útil é que permita consultar e utilizar informação sobre os elementos do edifício das várias especialidades que o compõem, como arquitetura, estrutura e instalações. Para isso é necessário que esta representação seja o mais fiel possível 
da realidade, facto conseguido através do conceito "digital twin", gémeo digital, aplicado na sua plenitude, ou seja, disponibilizando informação geométrica e não geométrica.

O trabalho que se apresenta diz respeito ao desenvolvimento da tecnologia Open BIM Systems, concebida para integrar os produtos dos fabricantes no fluxo de trabalho Open BIM, desenvolvido pela CYPE. Através de aplicações específicas e especializadas o utilizador é assistido na modelação e disposição dos elementos $3 \mathrm{D}$, com base em critérios de cálculo, verificações regulamentares e controlo de interações.

Neste trabalho, começa-se por apresentar um breve resumo do fluxo de trabalho Open BIM, no qual se integram os Open BIM Systems, fluxo este, suportado pela plataforma BIMserver.center e software CYPE, mas aberto a qualquer software BIM. Seguidamente apresenta-se a tecnologia Open BIM Systems, bem como o seu desenvolvimento a partir de dois objetivos específicos de utilização: por um lado os projetistas e por outro os fabricantes. Na parte final do trabalho apresentam-se dois casos de estudo de Open BIM Systems, aplicando a tecnologia concebida, dando primazia à metodologia de simulação através da utilização das aplicações de software desenvolvidas, Open BIM S3F Signs e Open BIM DAIKIN. Por fim, apresentam-se as conclusões evidenciando as potencialidades desta nova tecnologia.

\section{Fluxo de trabalho Open BIM}

Os estudos realizados permitiram concluir que o fluxo de trabalho Open BIM é único quando comparado com outros [1], pelo facto da plataforma BIMserver.center e do software CYPE se encontrarem integrados e ao mesmo tempo abertos a qualquer outro software BIM. O fluxo ganha especial vantagem na otimização dos processos que conduzem à obtenção e utilização do modelo federado do edifício, uma vez que para além do fluxo de trabalho proposto, o software CYPE abarca as várias especialidades do projeto de edifícios e cada aplicação permite por si só modelar, calcular e dimensionar, conduzindo também a uma diminuição dos recursos necessários.

A Figura 1 pretende ilustrar este fluxo de trabalho que inicia com a abertura do projeto na plataforma, pelo coordenador de projeto, bem como a criação da equipa de trabalho e as condições de acesso de cada colaborador.

Seguidamente o arquiteto partilha o modelo arquitetónico na plataforma BIMserver.center. $\mathrm{O}$ modelo arquitetónico pode ser gerado através de um programa de modelação de arquitetura ou da aplicação CYPE - IFC Builder, disponível gratuitamente na plataforma.

Com base no modelo arquitetónico, cada engenheiro cria o modelo inicial da sua especialidade, definindo e caracterizando os respetivos elementos. Depois, seleciona as funções de cálculo, dimensionamento e de verificações regulamentares, deste modo obtém automaticamente um modelo modificado resultante do processo de cálculo. O processo é iterativo, os elementos que constituem o modelo podem ser modificados e de novo verificados, até que todos se encontrem validados pelo engenheiro. Todo este processo, de modelação e cálculo, ocorre ao nível das aplicações do software CYPE. Desta forma obtém o modelo final e partilha-o na plataforma, ficando disponível para todos os projetistas da equipa de trabalho (arquitetos e engenheiros).

Cada projetista dispõe então do modelo federado, à medida que vai sendo construído, ou seja, à medida que cada um disponibiliza o seu próprio modelo na plataforma. Através do modelo federado é partilhada informação geométrica e não geométrica e são também partilhados documentos com peças escritas e desenhadas. 
O coordenador de projeto coordena as várias especialidades a partir da análise do modelo federado, localizado na plataforma, podendo utilizar a aplicação Open BIM Model Checker para identificação de interferências e colisões.

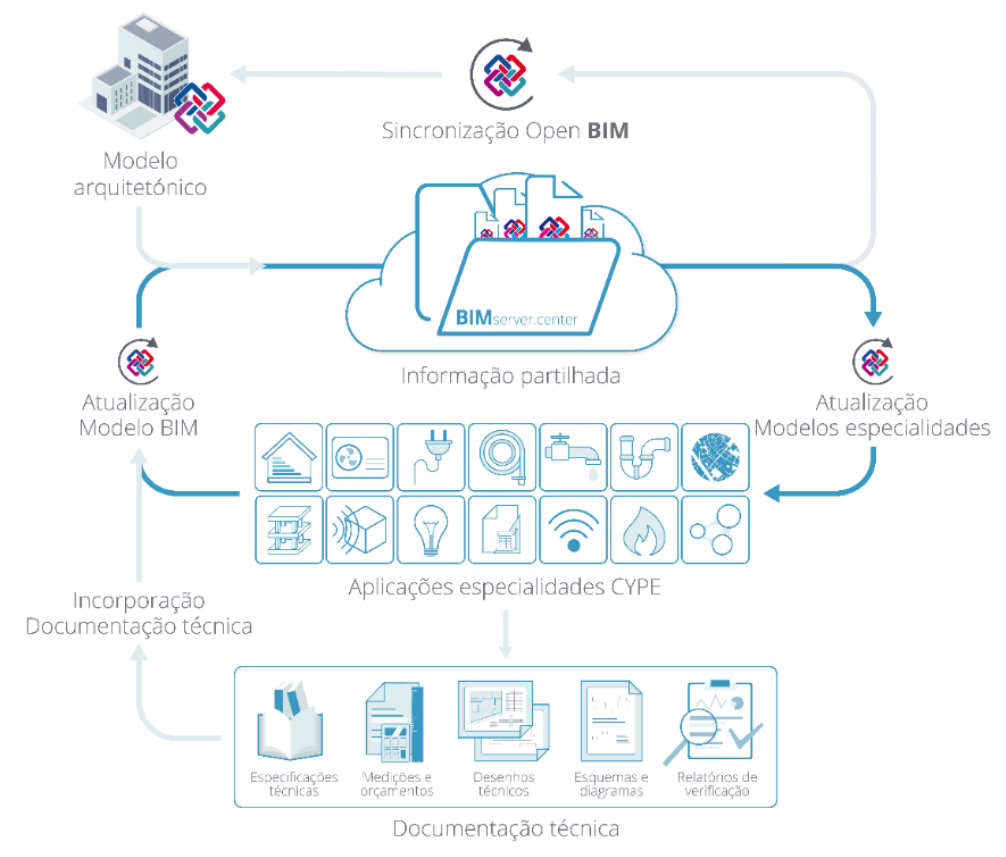

Figura 1: Fluxo de trabalho Open BIM.

\subsection{BIMserver.center}

O BIMserver.center [1] é uma plataforma colaborativa localizada na nuvem, destinada à partilha e troca de informação para o projeto de edifícios.

Apresenta características como: a possibilidade de definição da equipa de projeto, incluindo sistema de autorizações, coordenação e validação da informação; ligação direta e automática às aplicações das especialidades do projeto, através do formato aberto IFC (Industry Foundation Classes), incluindo a comunicação de resultados; partilha de informação geométrica e não geométrica e registo do historial de alterações; sincronização da informação partilhada das especialidades do projeto e obtenção do modelo federado; é local de encontro de entidades intervenientes no projeto.

A plataforma foi desenvolvida para permitir o acesso a partir do computador ou dispositivos móveis, como o tablet ou smartphone, através do seu Visualizador. As aplicações BIMserver.center AR e BIMserver.center VR permitem ainda consultar o modelo federado em realidade aumentada ou virtual, respetivamente.

\subsection{Software CYPE}

O software CYPE [2] abarca várias áreas como: modelação arquitetónica, análise estrutural, energética, acústica, MEP, estimativas orçamentais, medições, orçamentos, urbanismo e planeamento. A grande maioria das aplicações são de âmbito genérico, no entanto, algumas que foram criadas para marcas específicas de fabricantes são designadas por Open BIM Systems, sendo objeto do presente trabalho. Cada aplicação está munida de funções que permitem a sincronização com a plataforma a qualquer momento, através deste processo verifica-se a 
partilha do modelo federado, que se torna visível na própria aplicação, de acordo com o seu estado de avanço, sendo assim partilhada informação geométrica e não geométrica.

A grande maioria das aplicações encontra-se na Store do BIMserver.center, podendo ser descarregadas a partir daí. As aplicações Open BIM Systems são gratuitas e podem ser descarregadas e utilizadas livremente.

\section{Open BIM Systems}

Os Open BIM Systems são aplicações do software CYPE, tal como referido em 2.2, concebidas à medida para os fabricantes de materiais e equipamentos, destinadas à indústria da construção. No âmbito deste mesmo trabalho, foi feita uma análise com o objetivo de determinar o desempenho de objetos BIM disponíveis no mercado, tendo-se concluído que os objetos carecem de uma adequada definição dos atributos para o seu dimensionamento e/ou integração no sistema global, ou seja, nos projetos de especialidades de arquitetura e/ou engenharia. Se o BIM aspira verdadeiramente a gerar tecnologia que reduza custos e prazos, a definição dos objetos tem de ser algo mais do que estético ou publicitário, os objetos deverão ter a informação necessária e suficiente para poder simular o seu comportamento nos edifícios [3].

É aqui que nasce a ideia do projeto de conceção dos Open BIM Systems, ou seja, a necessidade de gerar uma aproximação fiel do objeto ao "gémeo digital", útil e indispensável para os programas de simulação, através do cálculo automático. A tecnologia dos Open BIM Systems trata os produtos não como objetos independentes, mas como objetos parametrizáveis, passíveis de serem dimensionados, ver Figura 7, e integrados num sistema que, por sua vez, se relaciona com outros sistemas partilhados na plataforma BIMserver.center. Esta tecnologia procura assim minimizar a lacuna assinalada.

No projeto de conceção dos Open BIM Systems foram identificadas especificações distintas daquelas que habitualmente se identificam na conceção das restantes aplicações do software CYPE, referem-se duas das principais:

- A necessidade de identificar a informação do fabricante, nomeadamente sobre os seus produtos, tanto geométrica como não geométrica;

- A necessidade de identificar especificações da aplicação, do ponto de vista do fabricante do produto e do projetista que a utilizará.

No primeiro caso, verificou-se que por regra o fabricante já dispõe de informação sobre os seus produtos em formato digital, esta existência permitirá acelerar o processo de conceção da aplicação e simultaneamente reduzir custos. Os objetos são transferidos do formato nativo para o formato STEP (Standard for the Exchange of Product model data), padrão internacional para a integração, apresentação e intercâmbio de dados de produtos industriais, e depois são incorporados, tratados e melhorados com vista à sua incorporação nas bibliotecas das aplicações Open BIM Systems. Este processo foi conseguido através de um "kernel" desenvolvido pela empresa C3D Labs.

No segundo caso, as especificações foram identificadas a partir de uma análise individualizada, baseada nas características de cada fabricante e nas suas necessidades particulares, bem como nas características e necessidades dos projetistas, e a resposta é a programação à medida para cada fabricante. Esta programação seguiu os padrões de "engenharia e arquitetura de software" 
da CYPE, dando cumprimento a três novos grupos de objetivos que se identificaram e enumeram seguidamente:

\subsection{Modelação 3D}

- as aplicações permitirão que um modelo tridimensional de cada elemento seja: gerado, colocado na sua posição exata, visualizado no modelo do edifício e partilhado no modelo federado;

- o modelo federado, incluindo os "digital twins" do fabricante, poderão ser visualizados em realidade aumentada e virtual.

\subsection{Integração Open BIM}

- cada aplicação será compatível com o restante software de modelação;

- $\quad$ as aplicações permitirão interações multidisciplinares;

- o modelo federado do projeto perdurará sem necessidade da plataforma nativa;

- será prestada ajuda na colocação dos elementos no modelo do edifício, através de funcionalidades do tipo "assistente";

- cada aplicação incluirá exclusivamente os produtos de um fabricante;

- $\quad$ as aplicações proporcionarão a prescrição dos produtos nos projetos.

\subsection{Serviços BIMserver.center}

- $\quad$ os Open BIM Systems terão impacto nos milhares de utilizadores do BIMserver.center;

- os fabricantes poderão monitorizar a utilização de cada produto através da área Business da plataforma BIMserver.center;

- os fabricantes terão acesso ao projeto, se este for autorizado pelo projetista, podendo propor as suas soluções;

- $\quad$ os Open BIM Systems serão disponibilizados gratuitamente na Store da plataforma BIM server.center.

Por ser do âmbito privado dos fabricantes, não se apresenta neste documento a resposta obtida, nos casos de estudo, aos três primeiros objetivos da secção 3.3, contudo, apresentam-se as várias fases incluídas no algoritmo desenvolvido, com vista ao alcance desses mesmos objetivos, Figura 2.

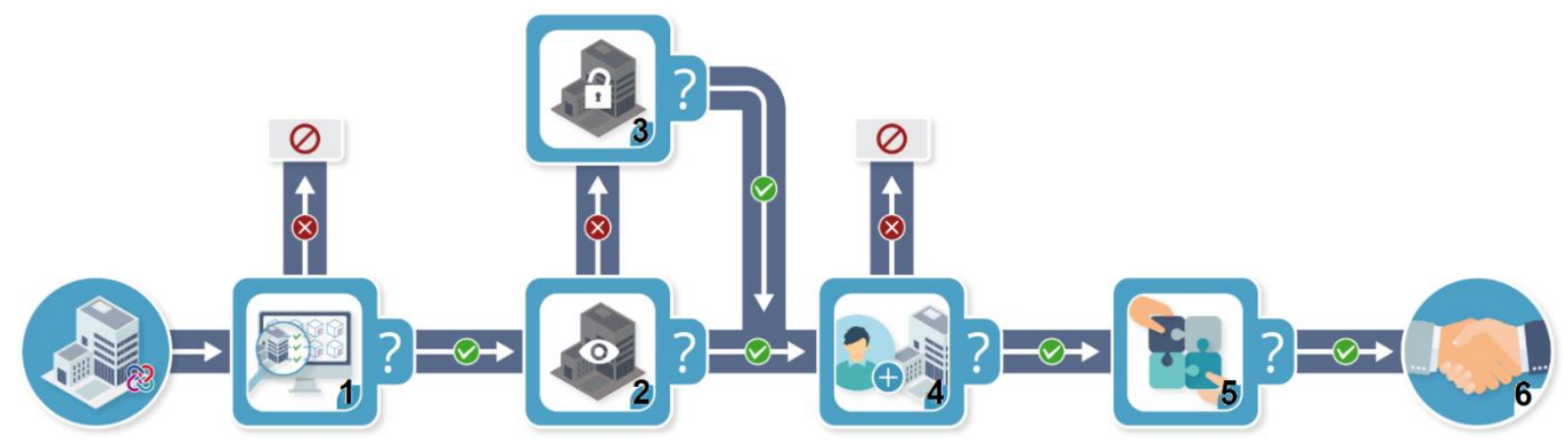

Figura 2: Algoritmo desenvolvido para a área Business. 
O algoritmo inicia com uma lista de especificações do "projeto objetivo", selecionadas pelo fabricante (por exemplo: edifícios destinados a hotelaria, com uma área superior a $5000 \mathrm{~m}^{2}$, localizados em Marrocos) e termina com a possível aceitação da proposta do fabricante por parte do projetista. O algoritmo é constituído pelas seguintes fases (questões):

- fase 1 - cumpre as especificações do projeto objetivo?

- fase 2 - o projeto é visível para o fabricante?

- fase 3 - como proprietário, aceita que o seu projeto esteja visível para o fabricante?

- fase 4 - foi aceite o pedido de participação?

- fase 5 - a proposta foi aceite?

- fase 6 - termina aqui a responsabilidade dos serviços prestados pelo BIMserver.center, esta fase corresponde à continuidade do processo, caso o dono de obra decida comprar os produtos do fabricante, e passa a ser gerida pelo fabricante.

Se na fase 3 o projetista mantiver o projeto invisível para o fabricante, o projetista pode desenvolver igualmente o seu projeto utilizando o Open BIM System do fabricante, dispensando apenas que este apresente a sua proposta de solução para o projeto em causa.

\section{Casos de estudo}

Foram desenvolvidos vários casos de estudo a fim de comprovar os resultados do projeto Open BIM Systems, nomeadamente ao nível do cumprimento dos objetivos anteriormente descritos. Neste trabalho apresentam-se dois casos de estudo: o Open BIM S3F Signs, concebido para um fabricante português, S3F Signs, que procura projeção, não só em Portugal, mas também a nível internacional, e o Open BIM DAIKIN, concebido para uma marca que já possui implantação internacional, a DAIKIN, mas que pretende continuar a reforçar a sua posição.

$\mathrm{O}$ primeiro é um sistema destinado à modelação de sinalética para edifícios. O segundo destinado ao cálculo e modelação de sistemas DAIKIN de VRV e Aerotermia. As aplicações encontram-se na Store do BIMserver.center e podem ser descarregadas gratuitamente, tal como referido anteriormente. Tal como referido na secção 3.3, nos casos de estudo que aqui se apresentam não é documentada a intervenção dos fabricantes por ser de âmbito privado.

\subsection{Open BIM S3F Signs}

Para o caso de estudo foi utilizado um edifício, de 5 pisos, destinado a escritórios, largamente estudado e usado como um dos exemplos padrão para testes ao software CYPE.

O processo iniciou-se através do procedimento descrito no capítulo 2 deste trabalho. Foi criado o projeto da obra no BIMserver.center, pelo coordenador de projeto, bem como a equipa de trabalho e as condições de acesso de cada colaborador. Foi partilhado o modelo arquitetónico na plataforma, gerado a partir da aplicação IFC Builder, este modelo pode observar-se na Figura 3 .

Seguidamente foram consideradas duas hipóteses: i) modelação da sinalética, no Open BIM S3F Signs, diretamente sobre o modelo arquitetónico e ii) modelação da sinalética, no Open BIM S3F Signs, sobre o modelo arquitetónico, mas respondendo aos requisitos resultantes da 
modelação realizada nas aplicações CYPEFIRE Design e Open BIM Accessibility (da biblioteca de software CYPE).

A aplicação CYPEFIRE Design, foi concebida para a modelação e cálculo de instalações de segurança contra incêndio (meios de proteção). A aplicação Open BIM Accessibility, foi concebida para a modelação e verificação de condições de acessibilidade de pessoas com mobilidade condicionada.

Para a hipótese i) foi criada a obra no Open BIM S3F Signs e sincronizado o modelo arquitetónico partilhado na plataforma. Procedeu-se de seguida à introdução dos sinais sobre cada uma das plantas, estes tornam-se automaticamente visíveis no modelo 3D, Figura 3.

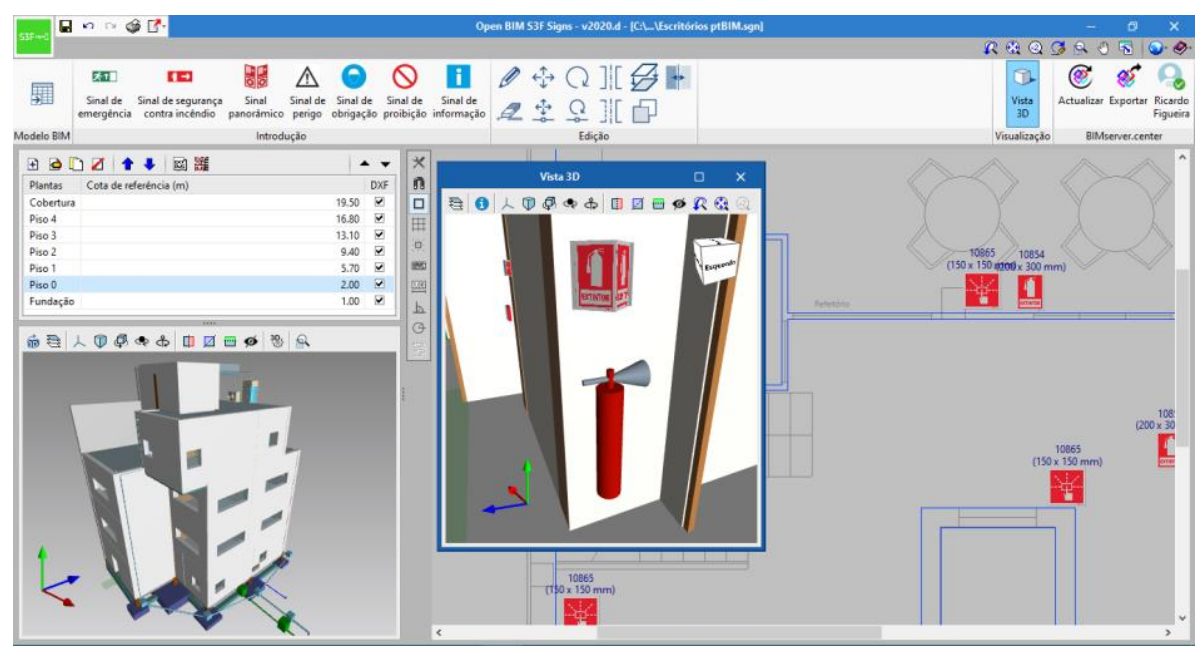

Figura 3: Ambiente de trabalho do Open BIM S3F Signs.

A Figura 3 é constituída pelo ambiente de trabalho da aplicação, dividido em funções comuns às aplicações CYPE (menu "Edição", "Visualização" e "BIMserver.center") e funções específicas da aplicação para a modelação da sinalética (menu "Introdução") e ainda na área de trabalho (plantas e modelo 3D). O menu "Introdução" contém os grupos de sinais que constituem a biblioteca S3F Signs, ou seja, o catálogo do fabricante. Cada grupo é constituído por vários modelos de sinais, sendo que a cada modelo corresponde uma série de dimensões, Figura 4.

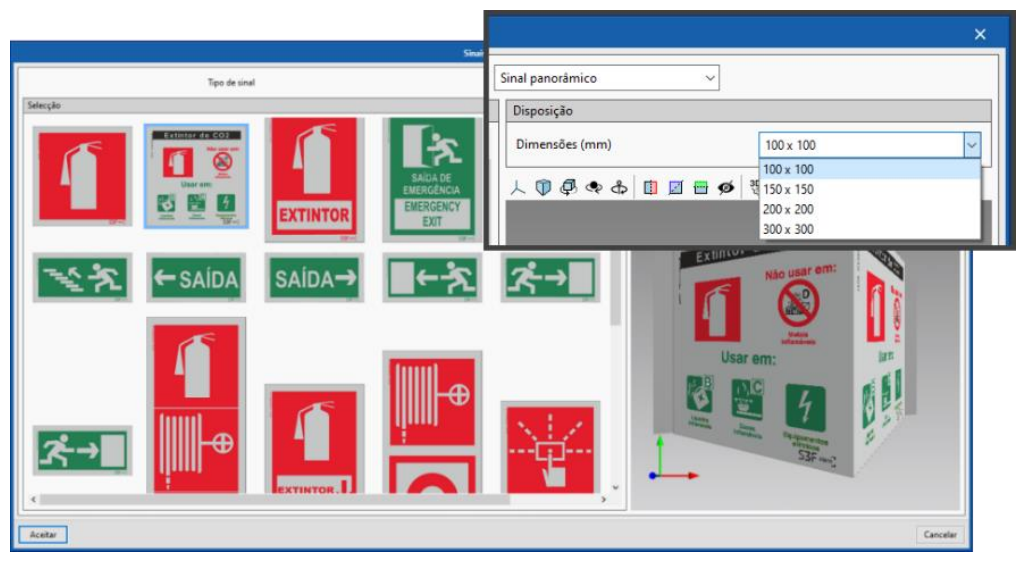

Figura 4: Exemplo de um grupo de sinais e da série de um sinal panorâmico. 
Terminada a modelação, foi possível partilhar o modelo IFC no BIMserver.center, incluindo o mapa de materiais, passando o modelo S3F Signs a ser integrado no modelo federado do projeto. O modelo federado passou então a conter os "digital twins" dos sinais a colocar em obra.

Para a hipótese ii) utilizou-se o procedimento descrito para a hipótese i), com a exceção de que para além da sincronização do modelo arquitetónico, foram também sincronizados os modelos do CYPEFIRE Design e Open BIM Accessibility, previamente definidos nas respetivas aplicações. Neste caso surgem automaticamente requisitos gerados por estas aplicações, ou seja, é identificada a necessidade de caracterizar sinais, materializados por um símbolo, tal como indicado na Figura 5 (imagem da esquerda). A partir deste momento procedeu-se à seleção do respetivo sinal na biblioteca do Open BIM S3F Signs, que substitui o símbolo previsto, Figura 5 (imagem da direita), repetindo-se o procedimento para todos os símbolos. Esta hipótese apresentou a vantagem de ser mais amigável e diminuir o erro humano quando comparada com a hipótese i) uma vez que os símbolos não caracterizados por sinais são assinalados através de um alerta.

O modelo IFC foi então partilhado na plataforma e o modelo federado passou a conter os "digital twins" dos sinais, tal como na hipótese i).
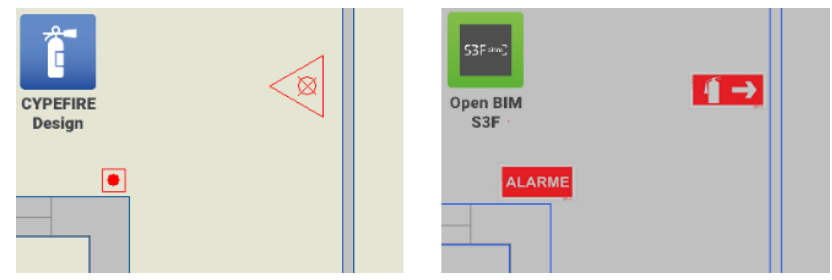

Figura 5: Pormenor de plantas CYPEFIRE Design e Open BIM S3F Signs.

O coordenador executou as suas tarefas de coordenação tanto em i) como em ii), através do Visualizador do BIMserver.center, do BIMserver.center AR e do BIMserver.center VR, utilizou também, para o apoiar, a aplicação Open BIM Model Checker que identifica automaticamente colisões e interferências. A Figura 6 apresenta uma imagem de um compartimento em realidade aumentada, onde são visíveis, entre outros, a sinalética e o ambiente exterior.

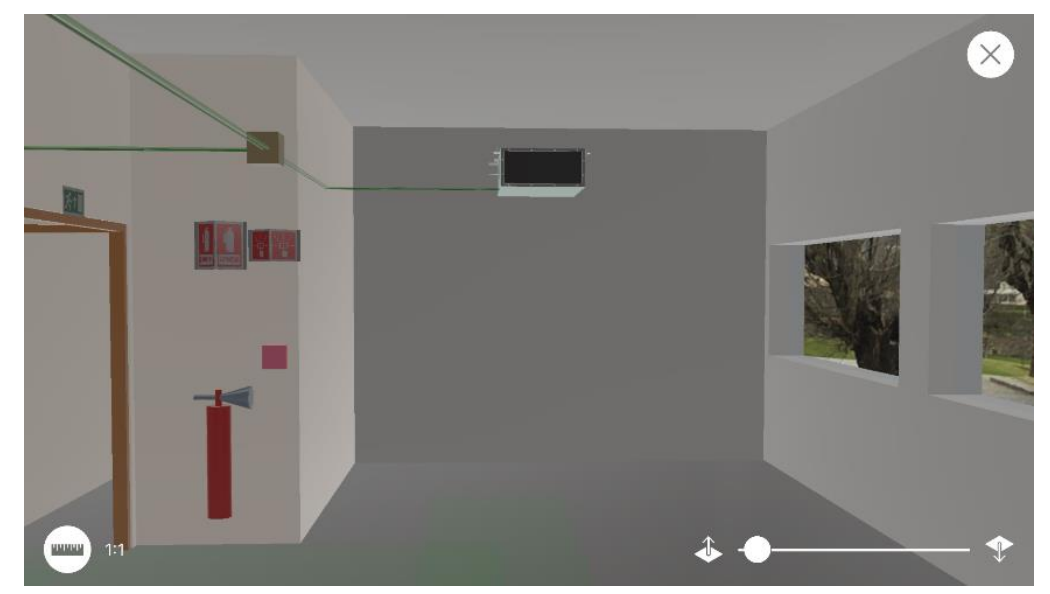

Figura 6: Pormenor do modelo federado (BIMserver.center AR). 


\subsection{Open BIM DAIKIN}

Esta aplicação distingue-se do Open BIM S3F Signs, uma vez que possui rotinas de cálculo, que permitem dimensionar todo o sistema desde os equipamentos exteriores e interiores de VRV e Aerotermia até às tubagens, ou seja, trata-se de dimensionar um sistema completo, para além da respetiva modelação, e não da simples modelação de objetos BIM.

Para a modelação são utilizadas as funções dos menus: "VRV", "Aerothermal" e "Pipes", cada menu é constituído pelos equipamentos da marca DAIKIN e cada equipamento é constituído pela sua série, com as respetivas características e requisitos de dimensionamento, Figura 7.

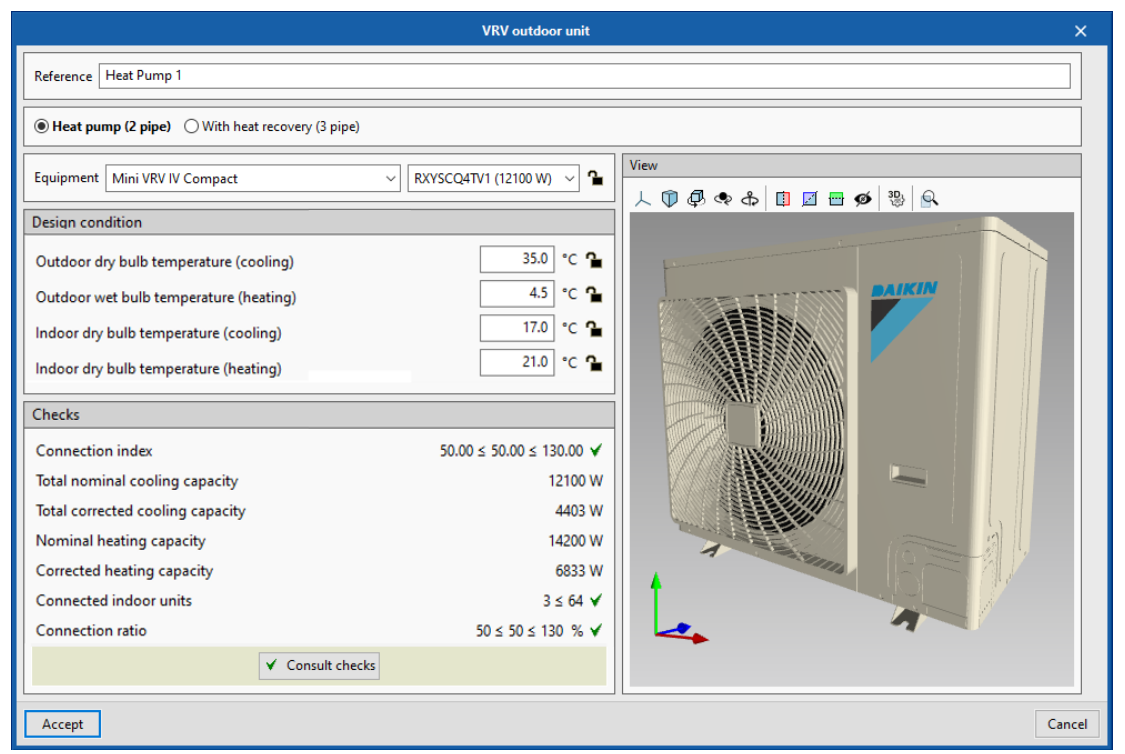

Figura 7: Unidade exterior de VRV da biblioteca da DAIKIN.

Para este caso de estudo, foi utilizado o edifício apresentado no caso de estudo anterior e foi realizado o procedimento referido em 4.1 até à criação da obra e sincronização do modelo arquitetónico.

Procedeu-se de seguida à modelação dos sistemas em cada uma das plantas, estes tornam-se automaticamente visíveis no modelo 3D. Terminada a modelação, cálculo e dimensionamento (processos automatizados e que ocorrem em simultâneo), o modelo IFC final foi então partilhado na plataforma e integrado no modelo federado, incluindo o relatório de cálculo e o mapa de materiais, tornando-se disponível para todos os colaboradores autorizados. O modelo pôde ser visualizado diretamente no BIMserver.center, através do BIMserver.center AR e do BIMserver.center VR. O modelo federado passou, então, a conter os "digital twins" dos sistemas a colocar em obra. A Figura 8 apresenta dois pormenores do modelo federado, um deles obtido através do Visualizador do BIMserver.center e outro, em realidade virtual, obtido através do BIMserver.center VR, em ambos é possível visualizar os equipamentos DAIKIN. 

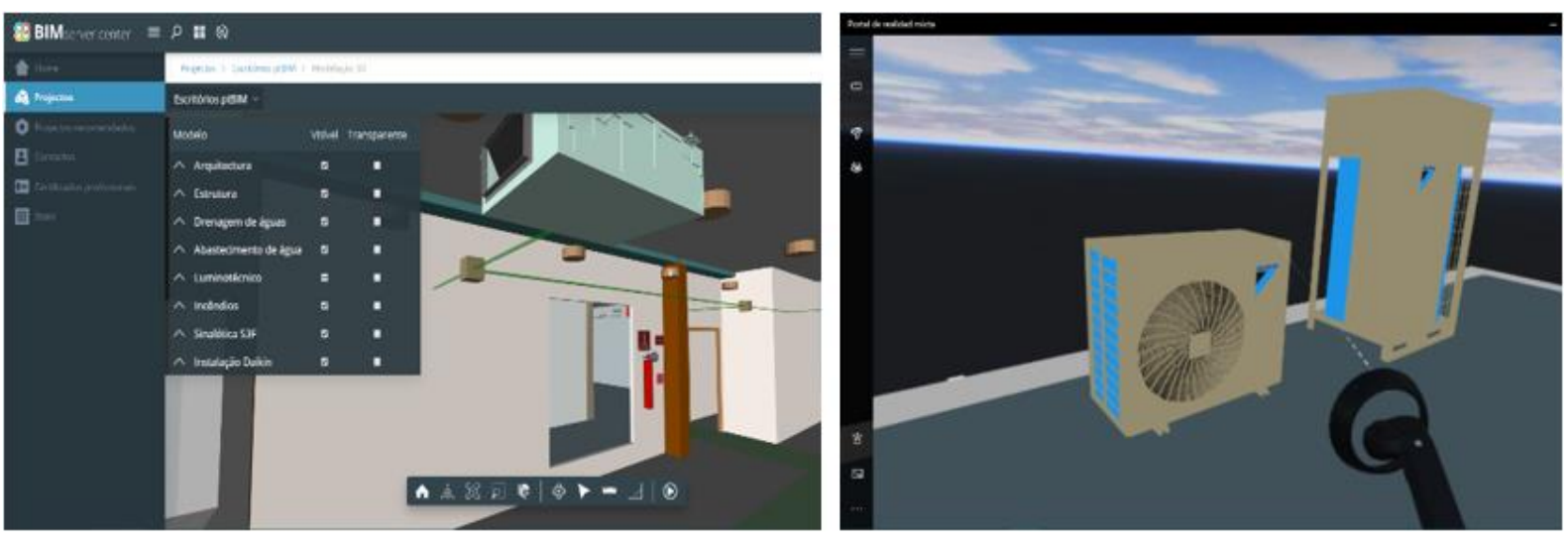

Figura 8: Pormenores do modelo federado (BIMserver.center e BIMserver.center VR).

\section{Conclusões}

O presente trabalho inicia com uma breve apresentação da plataforma colaborativa BIMserver.center, do software CYPE e do fluxo de trabalho Open BIM, tecnologias desenvolvidas no âmbito de projetos anteriores, mas que se revelam importantes para enquadramento dos Open BIM Systems.

A tecnologia Open BIM Systems, desenvolvida neste projeto, distingue-se dos objetos BIM existentes no mercado por ser um sistema que responde a especificações do projetista, nomeadamente ao nível da modelação 3D e integração no fluxo de trabalho Open BIM, e a especificações do fabricante, através dos serviços da área Business do BIMserver.center.

Os casos de estudo apresentados permitiram evidenciar o cumprimento dos objetivos definidos para o projeto Open BIM Systems, através das funcionalidades das aplicações Open BIM S3F Signs e Open BIM DAIKIN, nomeadamente modelar, calcular e dimensionar sistemas específicos de marcas de fabricantes, obter o respetivo modelo, em IFC, e partilhá-lo no modelo federado da obra, disponível na plataforma BIMserver.center, passando este a conter os "digital twins" dos materiais e equipamentos que serão colocados em obra.

\section{Referências}

[1] P. Assis, R. Figueira and P. Gilabert, "Plataforma Open BIM para o projeto de engenharia," in CONSTRUÇÃO 2018, Universidade do Porto, Porto, Portugal, 2018, pp. 740-749.

[2] P. Assis, R. Figueira and P. Gilabert, "Modelos BIM obtidos a partir do cálculo das especialidades do projeto de engenharia," in $2^{\circ}$ Congresso Português de Building Information Modelling, Instituto Superior Técnico, Lisboa, Portugal, 2018, pp. 509-598.

[3] A. Sistiaga, A. Candalija, P. Gilabert and B. Cantó, "Realidad aumentada y gemelos digitales," in V Congreso edifícios inteligentes, Grupo TECMARED, Madrid, Espanha, 2019, pp. 13-17. 